\title{
Stellar halos around Local Group galaxies
}

\author{
Alan W. McConnachie \\ NRC Herzberg, Dominion Astrophysical Observatory, 5071 West Saanich Road, Victoria, \\ British Columbia, Canada, V(E2E7 \\ email: alan.mcconnachie@nrc-cnrc.gc.ca
}

\begin{abstract}
The Local Group is now home to 102 known galaxies and candidates, with many new faint galaxies continuing to be discovered. The total stellar mass range spanned by this population covers a factor of close to a billion, from the faintest systems with stellar masses of order a few thousand to the Milky Way and Andromeda, with stellar masses of order $10^{11} M_{\odot}$. Here, I discuss the evidence for stellar halos surrounding Local Group galaxies spanning from dwarf scales (with the case of the Andromeda II dwarf spheroidal), though to intermediate mass systems (M33) and finishing with M31. Evidence of extended stellar populations and merging is seen across the luminosity function, indicating that the processes that lead to halo formation are common at all mass scales.
\end{abstract}

Keywords. galaxies: dwarf, galaxies: evolution, galaxies: formation, galaxies: general, galaxies: halos, Local Group, galaxies: stellar content, galaxies: structure

\section{Introduction}

As of September 2015, there are approximately 102 candidate Local Group galaxies known. Many of the newest discoveries are exceptionally faint, with literally only a handful of stars amenable to observations from which we can derive the properties of the stellar systems. It remains ambiguous how many of these new discoveries are dwarf galaxies, as opposed to globular clusters, tidal remains, or even asterisms. Figure 1 shows a luminosity function of all Local Group (candidate) galaxies. Red corresponds to the Milky Way and its satellite system $\left(D_{M W}<300 \mathrm{kpc}\right)$, blue corresponds to Andromeda and its satellites $\left(D_{A}<300 \mathrm{kpc}\right)$ and green corresponds to isolated galaxies in the Local Group (beyond 300kpc from Andromeda and the Milky Way but within the zero-velocity surface of the Local Group). Data for this luminosity function is given in McConnachie $(2012) \dagger$.

Figure 2 shows a map of the spatial distribution of red giant branch star candidates in the vicinity of Andromeda and Triangulum as observed by the Pan-Andromeda Archaeological Survey (PAndAS; McConnachie et al. 2009), color-coded by RGB color (redder RGB stars are probably more metal-rich in general than bluer RGB stars). Clearly, the halo of Andromeda is highly extended and highly substructured. However, whereas the majority of work on stellar halos is focused towards $L_{\star}$ galaxies like the Milky Way and Andromeda, it is worth highlighting that evidence for stellar halos can be found in galaxies at various positions in the luminosity function shown in Figure 1. Here, we discuss

$\dagger$ An updated version of this catalog is available online at http://www.astro.uvic.ca/ alan/Nearby_Dwarf_Database.html 


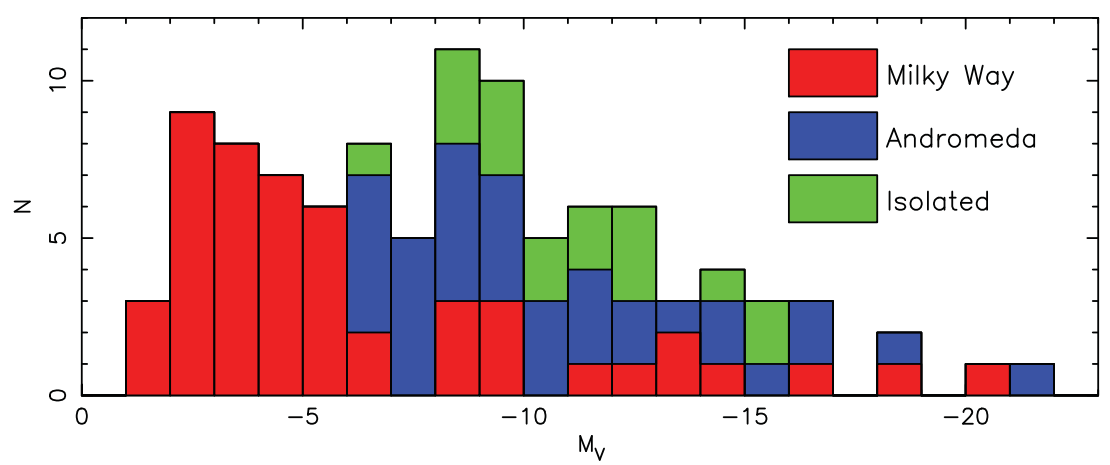

Figure 1. Luminosity function of all galaxies in the Local Group, using the September 2015 update from McConnachie (2012).

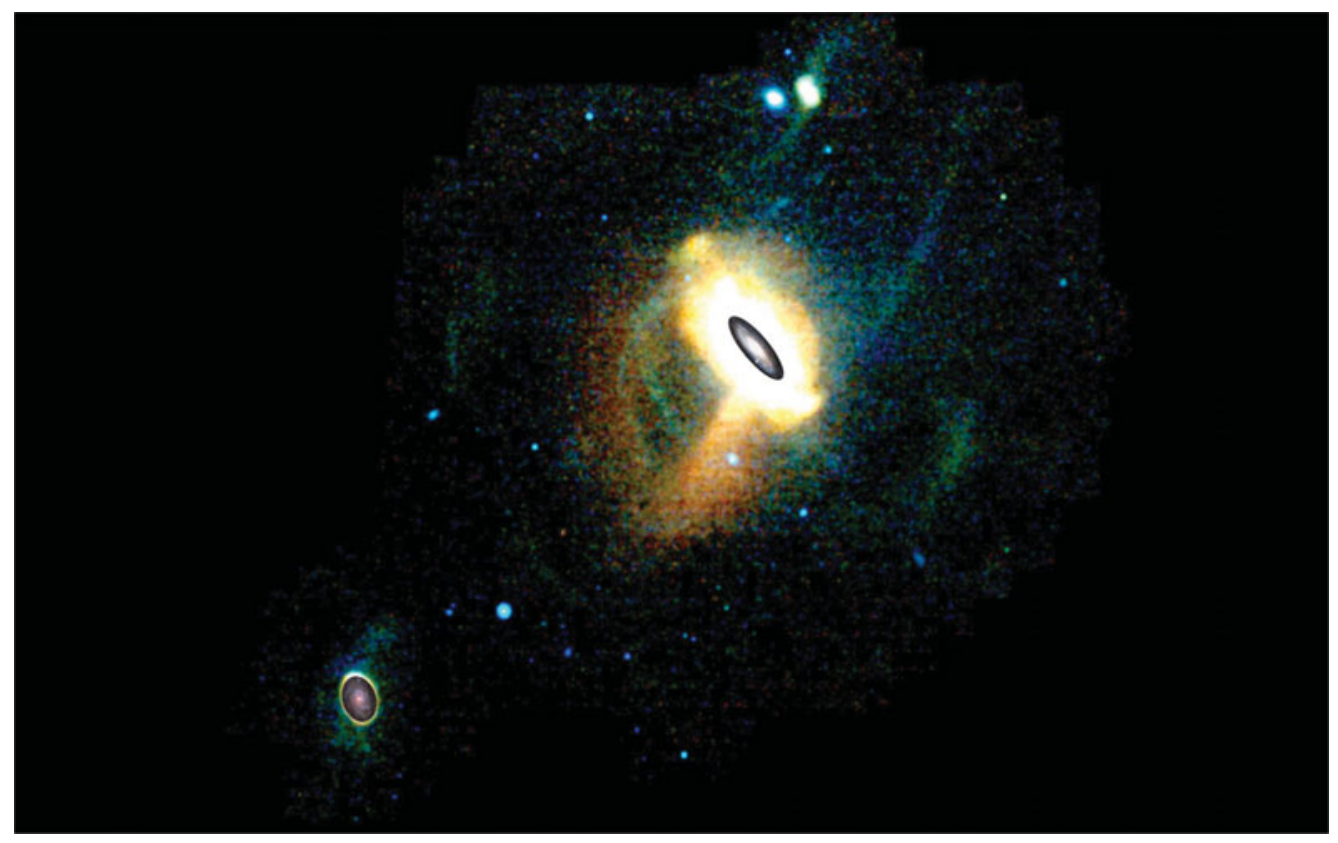

Figure 2. Spatial distribution of RGB stars surrounding Andromeda from PAndAS (McConnachie et al. 2009). Figure from Martin et al. (2013).

examples of stellar halos found in the Local Group across a range of luminosities. All the objects under discussion are visible in Figure 2.

\section{Stellar halos in dwarf galaxies: Andromeda II}

Using the Isaac Newton Telescope Wide Field Camera, McConnachie \& Irwin (2006) derive the surface brightness profiles of all the then-known dwarf spheroidal satellites of M31. These are all generally well described by King or exponential profiles, with the exception of Andromeda II. Andromeda II has a luminosity of $M_{V} \simeq-12.6$. For this galaxy, a significant discontinuity at $\sim 2$ arcmins means that a single profile that fits the majority of the radial profile grossly underestimates the number of stars at smaller radii. Subsequent follow-up work with the Subaru SuprimeCam (McConnachie et al. 2007) shows that, when split by stellar population, the structure of Andromeda II could 

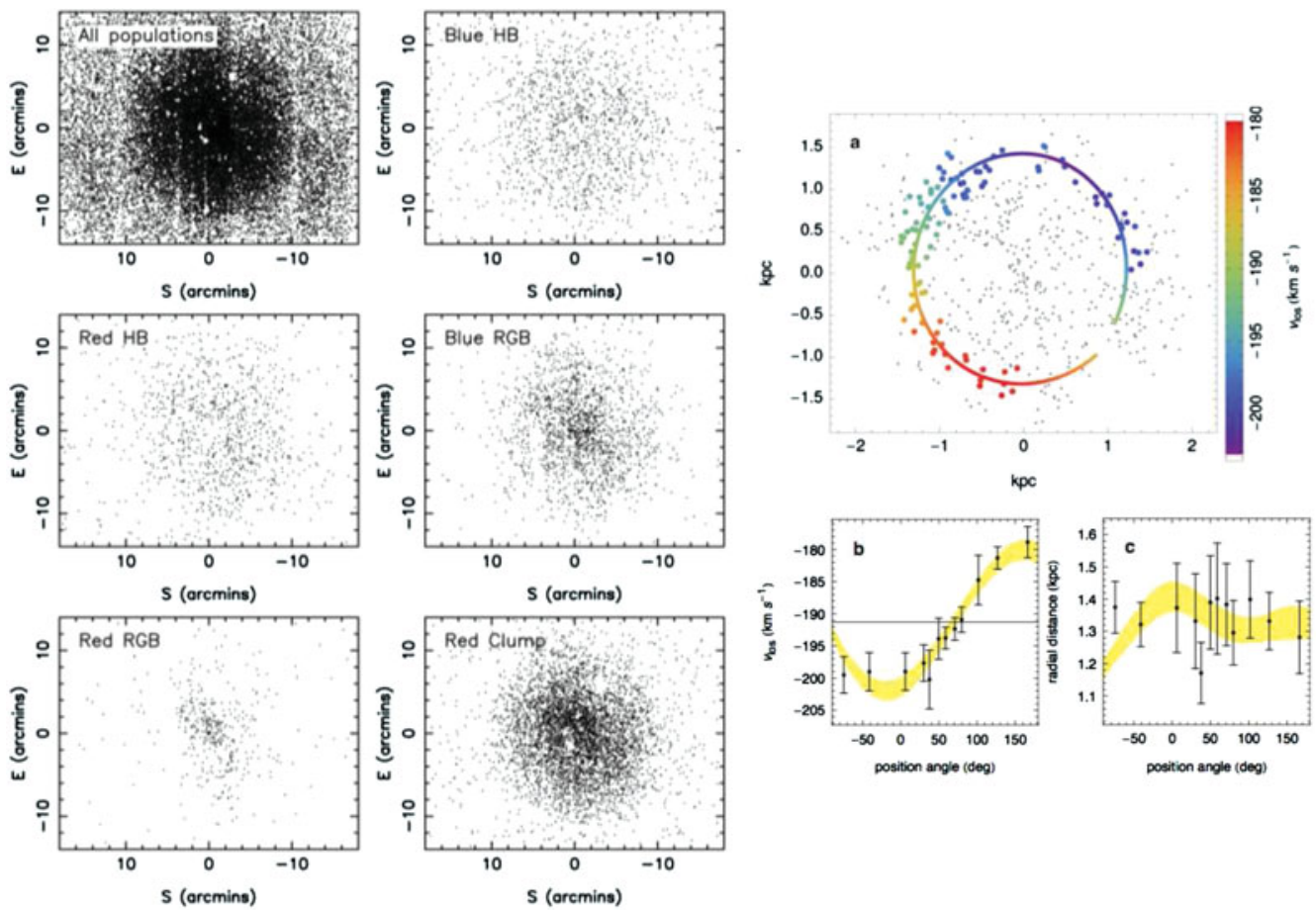

Figure 3. Left panels: stellar distribution of various stellar populations in Andromeda using Subaru/SuprimeCam (figure from McConnachie et al. 2007). Right panel: kinematics of stars in Andromeda II, highlighting a coherent "stream-like" structure in the outskirts of this galaxy. Figure from Amorisco et al. (2014)

vary dramatically depending on which tracer population was used. In particular, the horizontal branch population shows an extremely extended distribution, whereas redder RGB stars reveal a more centrally concentrated, exponential-style distribution. The left panels of Figure 3 show maps of Andromeda II using these different stellar populations as identified from the color-magnitude diagram.

One interpretation of these results is that Andromeda II has at least two distinct components to its structure, each with slightly different characteristic stellar populations, the more centrally concentrated of which is well traced by redder RGB stars, and the more extended of which is well traced by horizontal branch stars. However, while it is clear that Andromeda II does possess a spatially extended stellar component, its origin is unclear based only on the wide-field Subaru data. More recently, Amorisco et al. (2014) use a kinematic survey of Andromeda II by Ho et al. (2012) to identify a population of stars that have a kinematically distinct signature in Andromeda II and that trace a potential stellar stream in this dwarf galaxy (right panels of Figure 3). They conclude that Andromeda II has undergone a merger. When interpreted in the context of the earlier photometric work, Andromeda II shows evidence of a spatially extended secondary component whose origin is likely through a merger with a less luminous system, i.e., a stellar halo. Deep HST imaging of Andromeda II from Weisz et al. (2014) also points to a merger origin, since here the RGB can be seen to bifurcate, indicating at least two distinct populations, that perhaps formed originally in different systems. 


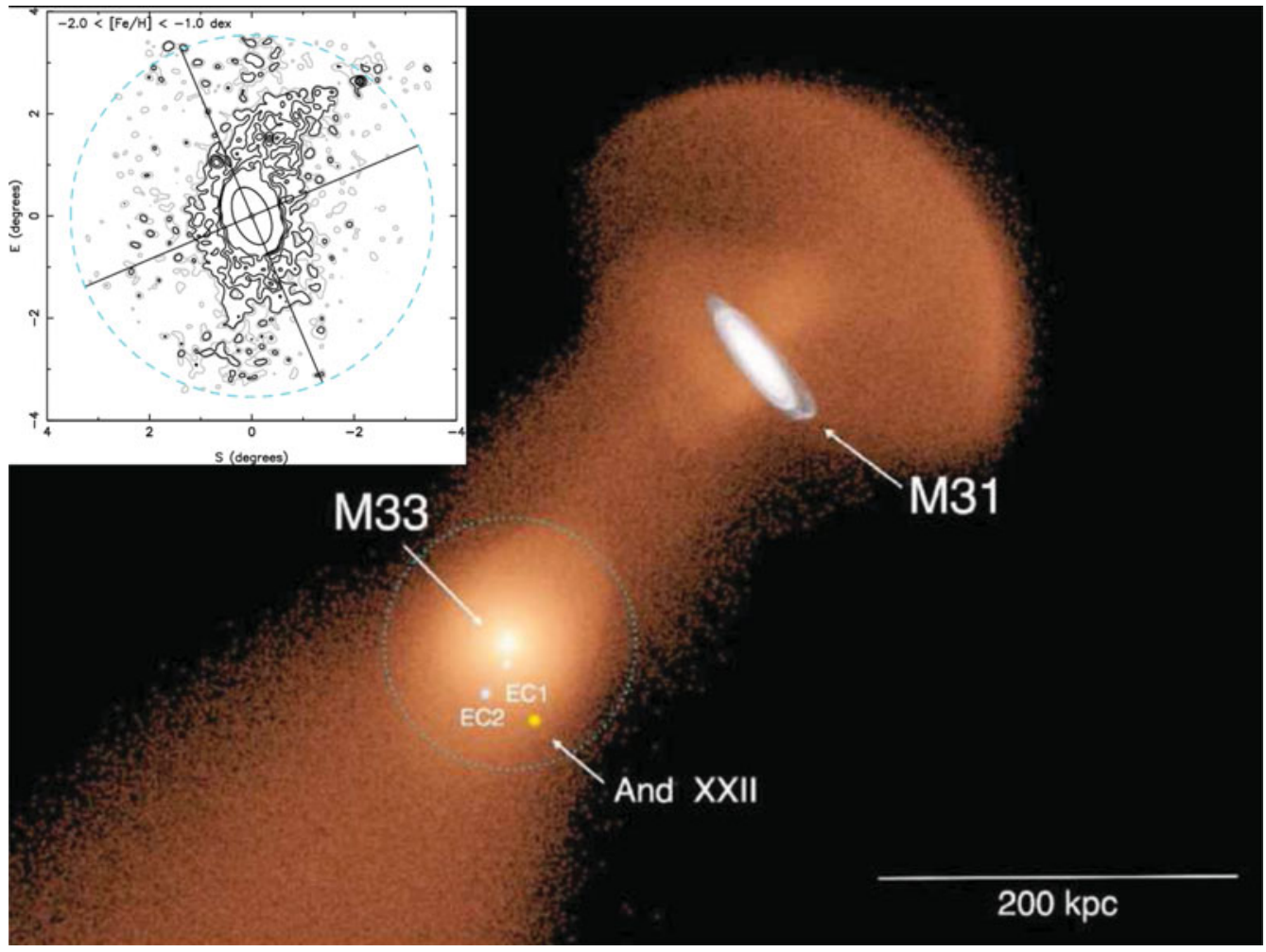

Figure 4. Inset panel: Distribution of metal poor RGB stars surrounding M33, showing a very large, stream-like distortion surrounding this galaxy. Figure from McConnachie et al. (2010). Large panel: dynamical model of M33 in orbit around M31, showing the potential stripping of the dark matter halo of M33 in its interaction with M31. Figure from Chapman et al. (2013).

\section{Stellar halos in sub-L $\star$ galaxies: M33}

M33 is the third most massive galaxy in the Local Group, but is still an order of magnitude less luminous than either M31 or the Milky Way. There have been several different claims to have detected a stellar halo in this galaxy over the years. Chandar et al. (2002) conducted an analysis of the star clusters in this galaxy and concluded that the oldest star clusters have distinct kinematics from the youngest clusters, and also from the HI disk. Their analysis suggested that the oldest clusters are consistent with having an 85-15\% halo-disk contribution. Subsequently, Sarajedini et al. (2006) show that the metallicitiy distribution of M33 as implied from the period of RR Lyrae stars has a bimodal distribution, and they attribute the metal-poor peak to a contribution from the stellar halo.

Both of these analyses are based on the study of fields within the main disk of M33, and the presence of an extended population at large radius (i.e., a stellar halo) had not been explicitly identified. McConnachie et al. (2006) conduct an analysis of the kinematics of two major axis fields surrounding M33 using Keck/DEIMOS located beyond the edge of the main disk $\left(>0.5^{\circ}\right)$. The majority of stars that they identify have kinematics consistent with the stellar disk, but they identify two additional features, one of which they attribute to a halo component, and another peak that they suggest could be some unidentified substructure. Subsequently, Ibata et al. (2007) used incomplete imaging using CFHT/MegaCam surrounding M33 to identify a significant population of stars at 


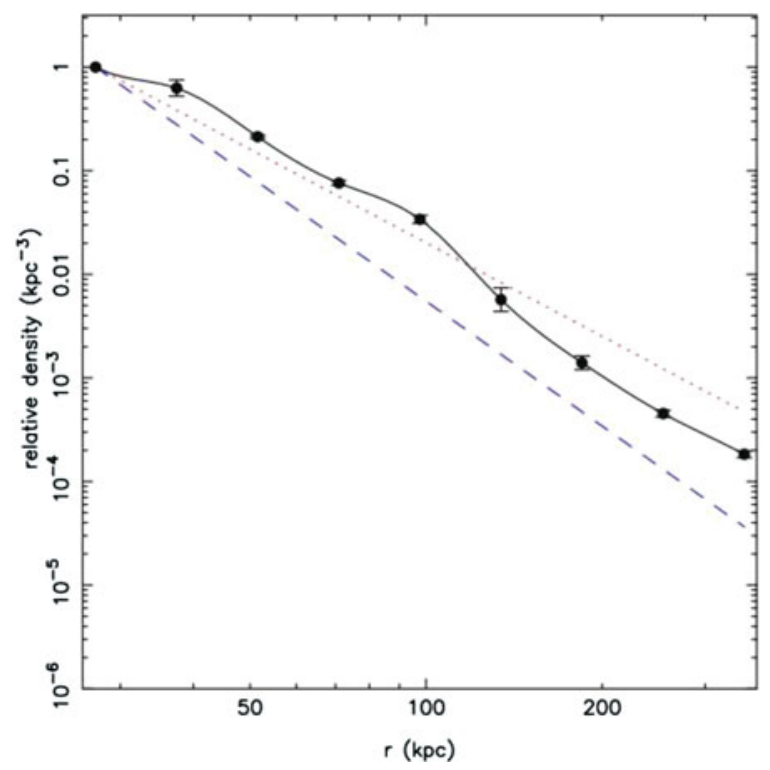

Figure 5. Derived profile of the "smooth" stellar halo of M31. Figure from Ibata et al. (2014).

distances of several degrees, confirming the apparent detection of a stellar halo around this galaxy.

However, the complete CFHT/MegaCam panorama of M33 was not completed until McConnachie et al. (2009), and it becomes immediately apparent that the stellar halo of M33 identified by Ibata et al. (2007) is not a typical, spheroidal-like population. The inset panel of Figure 4 is from McConnachie et al. (2010) and shows the distribution of RGB stars at several degrees from this galaxy. This population traces a very large, extended, "S-like" population that is fully consistent with what is expected by a galaxy that is undergoing tidal disruption in an orbit around a larger host. Dynamical modeling of an M31-M33 encounter in McConnachie et al. (2009) and Chapman et al. (2013; see Figure 4, main panel) suggests that a moderately-close fly-by of M33 by M31 should be enough to strip a large fraction of the dark matter halo, and presumably outer stellar populations, of M33. It is unclear if the material that is now observed is therefore the remnants of a previously extended, spheroidal halo-like population, or the stripped outskirts of the M33 stellar disk. Cockcroft et al. (2011) search for any remaining outer stellar populations in M33 away from the main debris, and find only tentative evidence for an underlying population. Having defined a stellar halo as a spatially-extended, outer stellar population, it is clear that M33 indeed possesses such a structure, but its formation mechanism could be quite different compared to other stellar halos, for example around M31.

\section{Stellar halos in $\mathrm{L} \star$ galaxies: Andromeda}

Figure 2 shows the extent and complexity of the stellar halo of M31, and this remains the best panoramic image of any stellar halo. There are large numbers of vast substructures and streams, and a significant number of dwarf galaxies (visible in Figure 2 as blue overdensities). Characterisation of this stellar halo is ongoing. Recently, Ibata et al. (2014) attempt to quantify the properties of the halo assuming that there is a component that is well described as a "smooth" population. Here, "smooth" implies no obvious substructure down to the surface-brightness limits of the data, and is a convenient way of 
characterising the population. Based on a masking procedure, they find that the fraction of the halo population that is smoothly distributed varies significantly as a function of metallicity. Overall, only $1 \%$ of the halo is in a smooth distribution, but at the most metal-poor end, over $60 \%$ of the stars appear smoothly distributed. Figure 5 shows the radial profile of the smooth M31 halo. It has a power-law distribution (with $\gamma=-3--4$ ) and extends to beyond 300kpc (deprojected). This is an extremely extended population that is also consistent with the very extended spatial distribution of satellite galaxies (Richardson et al. 2011). Indeed, it remains to be seen if simulated stellar halos are able to match this type of profile. A companion paper, detailing the statisitical properties of the substructure in M31, is in preparation (McConnachie et al. in preparation).

\section{Conclusions}

Stellar halos are rich environments for understanding the evolutionary and formation processes of galaxies. Merging is a key process by which at least some of the population is formed, both in terms of galaxies merging into the main system and being disrupted, with the remnants being identified with the stellar halo (e.g., Andromeda II and M31), and in terms of the main galaxy itself being disrupted (e.g., M33). Such processes occur in all luminosity regimes in the Local Group and are not confined to $L *$ galaxies.

\section{References}

Amorisco, N. C., Evans, N. W., \& van de Ven, G. 2014, Nature, 507, 335

Chandar, R., Bianchi, L., Ford, H. C., \& Sarajedini, A. 2002, ApJ, 564, 712

Chapman, S. C., Widrow, L., Collins, M. L. M., et al. 2013, MNRAS, 430, 37

Cockcroft, R., McConnachie, A. W., Harris, W. E., et al. 2013, MNRAS, 428, 1248

Ho, N., Geha, M., Munoz, R. R., et al. 2012, ApJ, 758, 124

Ibata, R. A., Lewis, G. F., McConnachie, A. W., et al. 2014, ApJ, 780, 128

Ibata, R., Martin, N. F., Irwin, M., et al. 2007, ApJ, 671, 1591

Martin, N. F., Ibata, R. A., McConnachie, A. W., et al. 2013, Apj, 776, 80

McConnachie, A. W. 2012, AJ, 144, 4

McConnachie, A. W., Chapman, S. C., Ibata, R. A., et al. 2006, ApJ, 647, L25

McConnachie, A. W., Ferguson, A. M. N., Irwin, M. J., et al. 2010, ApJ, 723, 1038

McConnachie, A. W., Arimoto, N., \& Irwin, M. 2007, MNRAS, 379, 379

McConnachie, A. W. \& Irwin, M. J. 2006, MNRAS, 365, 1263

McConnachie, A. W., Irwin, M. J., Ibata, R. A., et al. 2009, Nature, 461, 66

Richardson, J. C., Irwin, M. J., McConnachie, A. W., et al. 2011, ApJ, 732, 76

Sarajedini, A., Barker, M. K., Geisler, D., Harding, P., \& Schommer, R. 2006, AJ, 132, 1361

Weisz, D. R., Skillman, E. D., Hidalgo, S. L., et al. 2014, ApJ, 789, 24 